\title{
Longitudinal Progression of Dysphagia in Huntington's Disease and Dentatorubral-Pallidoluysian Atrophy: $\geq 10$ Years Retrospection in Twelve Patients
}

\author{
Hiroyuki Todo*1, Kengo Hasegawa ${ }^{2}$, Keisuke Nishimoto ${ }^{1}$, Katsuya Nishida ${ }^{1}$, Naonobu Futamura ${ }^{1}$ and Itaru $^{*}$ \\ Funakawa $^{1}$ \\ ${ }^{1}$ Department of Neurology, National Hospital Organization Hyogo-Chuo National Hospital, Japan \\ ${ }^{2}$ Department of Rehabilitation, National Hospital Organization Hyogo-Chuo National Hospital, Japan
}

Received: 眥: September 20, 2018; Published: 眥: October 05, 2018

*Corresponding author: Hiroyuki Todo, Department of Neurology, National Hospital Organization Hyogo-Chuo National Hospital, Japan

Keywords: Dentatorubral Pallidoluysian Atrophy; Dysphagia; Huntington's Disease; Videofluorography

Abbreviations: ADL: Activity in daily lives; DRPLA: Dentatorubral-Pallidoluysian Atrophy; HD: Huntington's Disease; ND: No data; PEG: Percutaneous Endoscopic Gastrostomy

\section{Introduction}

Huntington's disease (HD) and Dentatorubral-pallidoluysian atrophy (DRPLA) are progressive and currently incurable neurodegenerative diseases which shares common features such as choreic involuntary movements, dementia, psychosis, and pathologic expansion of triplet nucleotide repeats in the causative genes [1,2]. In HD, the prevalence of dysphagia is approximately $30 \%$ and several reports have been examined the features [3-5]. In DRPLA, dysphagia can be clinically challenging considering a report that approximately $50 \%$ of the DRPLA patients decease by aspiration pneumonia [6]. However, the data is scarce about the natural course of dysphagia in both HD and DRPLA in long term. Thereby, we examined the progression to clarify the features and to seek clues for potential treatments.

\section{Materials and Methods}

We performed a retrospective chart-review of patients with HD $(\mathrm{N}=7)$ and DRPLA $(\mathrm{N}=5)$ whose swallowing function was examined at least once in our institution. The diagnosis of HD was made by simultaneous presence of following three features;

a) Triad of movement abnormality (i.e. involuntary movement, especially chorea, and/or bradykinesia), cognitive dysfunction, and psychiatric symptoms,

b) Atrophy of caudate in brain imaging, and

c) Pathologic expansion of CAG repeats in HTT in their own or their families; if gene testing not done, presence of both (a) and (b) in their family members. DRPLA was diagnosed by;

a) characteristic neurological symptoms/signs (e.g. chorea, epilepsy, ataxia, and psyhosis),

b) atrophy of brainstem and/or cerebellum, with or without leukoencephalopathy in brain imaging, and

c) mutation of ATN1 or family history, in the same way as HD above.

The chart-review was concluded if the patients deceased, transferred to other institutions, or received percutaneous endoscopic gastrostomy (PEG). The onset of disease was defined as the initiation of movement abnormality, excluding the start of congnitive-psychiatric symptoms or epilepsy. The level of nutritional modification was reviewed to express the severity of dysphagia in the following five stages (the mildest: I, the severest: V); (I) unmodified, (II) partially modified (e.g. particular foods were minced), (III) pasted, (IV) high-caloric jelly, and (V) enteral feeding via PEG. The activity in daily lives (ADL), when they became wheelchair-bound and/or bedridden, was reviewed to express the systemic motor function. The result of video fluorography (VF), using liquid barium, was also reviewed. Owing to the small sample size, the continuous variables were expressed in median (range).

\section{Results}

In the background of HD (case 1-7) and DRPLA (case 8-12), the age at disease onset was 49 (26-57) and 27(10-38) year-old 
(yo), the disease duration was 19 (10-29) and 17 (12-25) year in the last examination, respectively (Table 1 ). All had involuntary movements, prevalently chorea. For the preceding non-motor symptoms, case 5 had cognitive decline and case 8,10 , and 12 had epilepsy. As the endpoints, case 2 and 12 deceased, case 3 transferred to another institution, case $4,7,8,10$, and 11 received PEG, and the rest survived by oral intake. If the data was missing or the test was not done, it was described as no data (ND), and

Table 1: Patient profiles and time course in disease progression.

\begin{tabular}{|c|c|c|c|c|c|c|c|c|c|c|c|c|}
\hline \multirow{2}{*}{$\begin{array}{c}\text { Diagnosis } \\
\text { Case No. }\end{array}$} & \multicolumn{7}{|c|}{ HD } & \multicolumn{5}{|c|}{ DRPLA } \\
\hline & 1 & 2 & 3 & 4 & 5 & 6 & 7 & 8 & 9 & 10 & 11 & 12 \\
\hline \multicolumn{13}{|c|}{ Clinical profiles } \\
\hline Age at last exam (yo) & 58 & 74 & 77 & 45 & 57 & 64 & 68 & 48 & 46 & 27 & 54 & 50 \\
\hline Sexuality & M & $\mathrm{F}$ & $\mathrm{F}$ & $\mathrm{F}$ & $\mathrm{F}$ & M & $\mathrm{F}$ & $M$ & $\mathrm{~F}$ & M & $\mathrm{F}$ & M \\
\hline Disease onset (yo) & 29 & 53 & 57 & 26 & 47 & 54 & 49 & 23 & 27 & 10 & 38 & 38 \\
\hline Triplet repeats No. & ND & 42 & 48 & Exp & ND & Exp & Exp & Exp & ND & 65 & ND & ND \\
\hline Family history & + & - & - & + & + & - & - & + & + & + & + & + \\
\hline \multicolumn{13}{|c|}{ Decline of swallowing function (years after the disease onset) } \\
\hline Partially modified food & 18 & 13 & ND & ND & - & ND & ND & ND & 13 & ND & 13 & ND \\
\hline Pasted food & 19 & - & 15 & ND & - & ND & ND & 21 & - & ND & 13 & 10 \\
\hline Jelly & 20 & - & - & ND & - & 10 & ND & 23 & - & ND & - & 12 \\
\hline Enteral feeding (PEG) & - & - & - & 10 & - & - & 15 & 24 & - & 14 & $14^{*}$ & - \\
\hline \multicolumn{13}{|c|}{ Decline of ADL (years after the disease onset) } \\
\hline Wheelchair-bound & 22 & 9 & 15 & ND & 8 & $10 \#$ & ND & 17 & 10 & 10 & 10 & 11 \\
\hline Bedridden & 22 & 14 & 18 & 11 & - & - & 15 & 21 & 10 & - & $14^{*}$ & - \\
\hline \multicolumn{13}{|c|}{ Abnormality in videofluorography } \\
\hline Time after onset (years) & ND & 13 & 16 & ND & ND & 10 & ND & 22 & ND & 13 & 13 & 8 \\
\hline Mastication & ND & + & - & ND & ND & + & ND & + & ND & - & - & - \\
\hline Swallowing whole & ND & + & + & ND & ND & - & ND & + & ND & - & + & + \\
\hline Oropharyngeal transfer & ND & + & - & ND & ND & - & ND & - & ND & - & - & - \\
\hline Swallowing reflex & ND & - & - & ND & ND & + & ND & + & ND & - & - & + \\
\hline Aspiration & ND & - & - & ND & ND & - & ND & + & ND & + & - & - \\
\hline Residue & ND & + & + & ND & ND & + & ND & + & ND & + & - & - \\
\hline
\end{tabular}

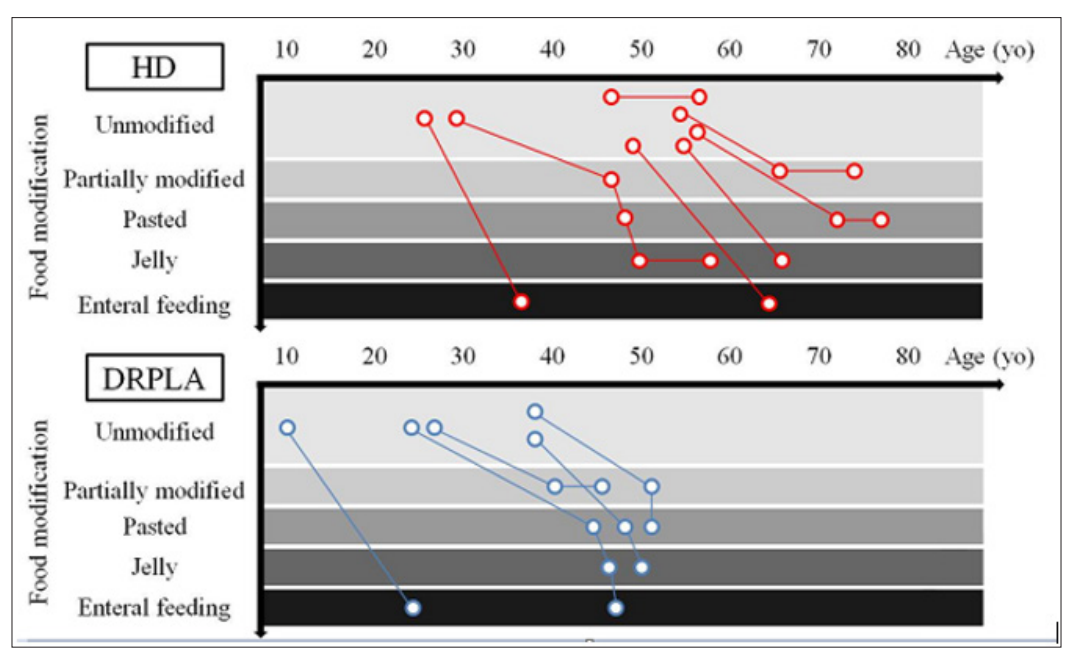

Figure 1: Progression of dysphagia in HD and DRPLA. 
Plotting of longitudinal decline in swallowing function (Figure 1) suggested that younger disease onset and having DRPLA are potential risk factors of poorer functional prognosis, although the small sample size and absence of young-onset HD and elderly-onset DRPLA were undesirable for statistical analysis. The decline in ADL due to disease progression, with exception of case 6 who became wheelchair-bound by spinal compressive fracture (annotated by \# in Table 1), seemed accompanying to the dysphagic progression with the lag within \pm 4 years. VF was evaluatable in 3 HD patients and 4 DRPLA patients, showing that insufficient mastication, impaired oropharyngeal transfer, delay in swallowing reflex, and aspiration in a certain number of patients. Swallowing whole foods (positivity: $66 \%$ in HD and $75 \%$ in DRPLA), and pharyngeal residue (positivity: $100 \%$ in HD and $50 \%$ in DRPLA) had relatively high prevalence in both groups. Notably, only case 10 experienced positional change from sitting position to reclining position in $\mathrm{VF}$ (Figure 2). The result was disappearance of aspiration (penetration of balium into the trachea: arrowhead) and pharyngeal residue (residue of balium in the pyriform sinus: arrow), as the fixation of his head and neck against choreic movement was ameliorated.

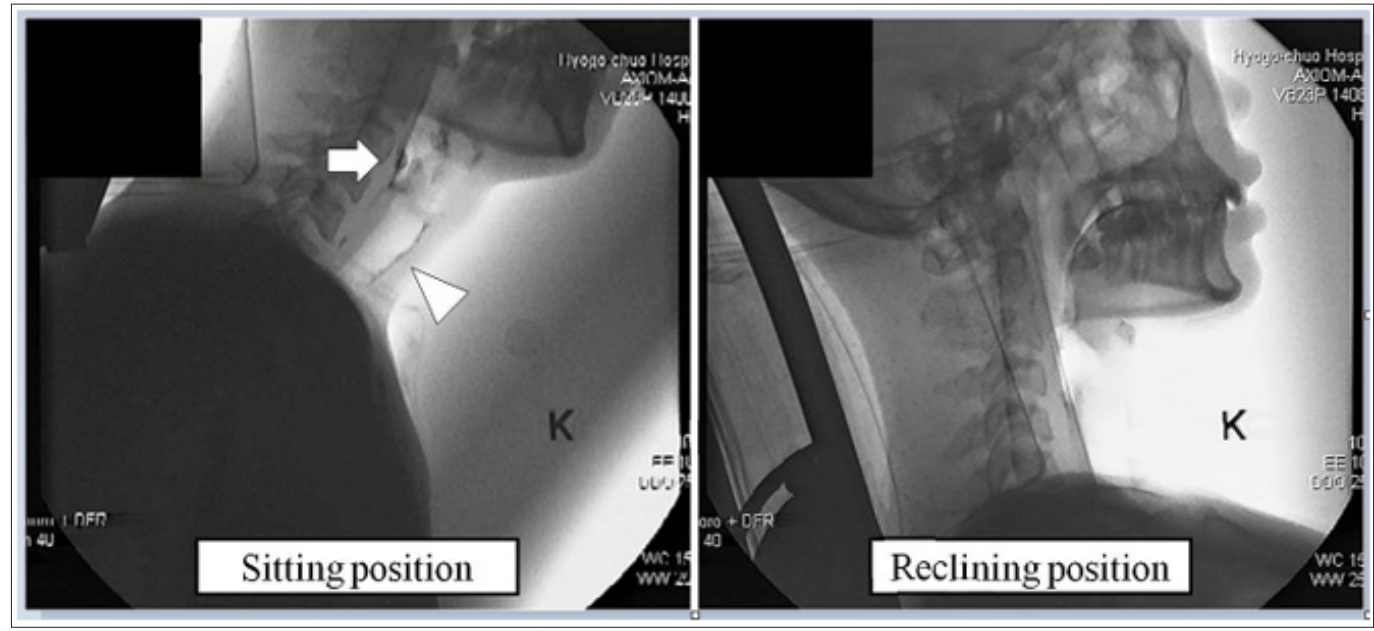

Figure 2: Positional adjustment of case 10.

\section{Discussion and Conclusion}

We reported the longitudinal progression of dysphagia in HD and DRPLA with $\geq 10$ years observation. Owing to the retrospective study design, the workups (e.g. gene testing and VF) were not thorough, furthermore the sample size, absence of age-matching in HD and DRPLA, and lack of healthy control were negative factors for precise statistical analysis. However, we found several suggestions from the data. In the early phase, dysphagia of HD and DRPLA was not prominent, considering amyotrophic lateral sclerosis often impose the patients to receive enteral feeding in 2-3 years [7]. However, when the duration of HD and DRPLA was $\geq 10$ years, dysphagia was no more ignorable considering that approximately $30 \%$ in HD and $40 \%$ in DRPLA of our patients received PEG. It seemed plausible that being young-onset and having DRPLA were potential risk factors of rapid progression in dysphagia, since younger onset are reported to be associated with severer phenotype in both HD and DRPLA (often by longer triplet expansion), and epilepsy and mental retardation in DRPLA can negatively influence the swallowing as well as the motor abnormality $[1,2,6]$. The seemingly positive correlation of swallowing function and ADL was consistent with the previous report of HD, while we could not find adequate reports of DRPLA [3].

It was also suggested that particular patients with unstable head and neck due to involuntary movements, as our case 10, can be benefited by reclining position. However, the significance of positional adjustment in HD and DRPLA is not established [4]. Rather, considering HD with rigid-bradykinetic phenotype can need verticality for smooth transfer of foods utilizing gravity, reclining position can interfere food transfer in these phenotypes [8]. In conclusion, approximately $30-40 \%$ of patients with HD and DRPLA had to receive enteral feeding by the disease progression in $\geq 10$ years from the onset of motor symptoms. The progression of dysphagia could be correlated to systemic motor function. Young onset and DRPLA could be risk factors of rapid decline in the swallowing function. However, further investigations are required owing to the limitations such as small sample size, lack of agematching in HD and DRPLA group, absence of controls, incomplete data of triplet repeat number, possible influence of haloperidol, and disunity of workup including VF.

\section{Acknowledgments}

The authors have no financial disclosure to report. This research did not receive any specific grant from funding agencies.

\section{References}

1. Pandey M, Rajamma U (2018) Huntington's disease: the coming of age. J Genet 97(3): 649-964.

2. Veneziano L, Frontali M, DRPLA (2016) GeneReviews® [Internet]. Seattle (WA): University of Washington, Seattle 1993-2018.

3. De Tommaso M, Nuzzi A, Dellomonaco AR, Sciruicchio V, Serpino C, et al. (2015) Dysphagia in Huntington's Disease: Correlation with Clinical Features. Eur Neurol 74(1-2): 49-53. 
4. Heemskerk AW, Roos RA (2011) Dysphagia in Huntington's disease: a review. Dysphagia 26(1): 62-66.

5. Hamilton A, Heemskerk AW, Loucas M, Twiston Davies R, Matheson KY, et al. (2012) Oral feeding in Huntington's disease: a guideline document for speech and language therapists. Neurodegen Dis Manage 2(1): 4553.

6. Hasegawa A, Ikeuchi T, Koike R, Matsubara N, Tsuchiya M, et al. (2010) Long-term disability and prognosis in dentatorubral-pallidoluysian

ISSN: 2574-1241

DOI: 10.26717/BJSTR.2018.09.001833

Hiroyuki Todo. Biomed J Sci \& Tech Res

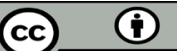

This work is licensed under Creative Commons Attribution 4.0 License

Submission Link: https://biomedres.us/submit-manuscript.php atrophy: a correlation with CAG repeat length. Mov Disord 25(11): 1694-700.

7. Spataro R, Ficano L, Piccoli F, La Bella V (2011) Percutaneous endoscopic gastrostomy in amyotrophic lateral sclerosis: effect on survival. J Neurol Sci 304(1-2): 44-48.

8. Kagel MC, Leopold NA (1992) Dysphagia in Huntington's disease: a 16year retrospective. (1992) Dysphagia 7(2): 106-114.

$\begin{array}{ll}\text { BIOMEDICAL } & \text { Assets of Publishing with us } \\ \text { RESEARCHES } & \text { - Global archiving of articles } \\ & \text { - Immediate, unrestricted online access } \\ & \text { - Rigorous Peer Review Process } \\ & \text { - Authors Retain Copyrights }\end{array}$

